\title{
Recent progress in carotenoid and retinoid synthesis
}

\author{
Masayoshi Ito, Yumiko Yamano, Sanae Sumiya and Akimori Wada \\ Kobe Women's College of Pharmacy \\ 4-19-1, Motoyamakita-machi, Higashinada-ku, Kobe 658, Japan
}

\begin{abstract}
Photoisomerization of peridinin and its related sulfone gave the novel $6 S$ allenic isomers. (6S)-Peridinin was synthesized in an optically active form. The first total synthesis of optically active fucoxanthin was accomplished starting from the readily available $(4 R, 6 R)$-4-hydroxy-2,2,6-trimethylcyclohexanone by the application of the rearrangement of $\alpha$-acetylenic alcohols to $\alpha, \beta$-unsaturated carbonyl compounds by silylvanadate catalyst followed by iodine-catalyzed isomerization. Recent work on the synthesis of bicyclic retinal analogues is also described.
\end{abstract}

In connection with studies on photosynthesis, further interest has again been centered around the two major allenic carotenoids, fucoxanthin 1 and peridinin 2 (Scheme 1) (ref. 1), which function as light-harvesting pigments for photosynthesis in the sea and have, in vivo, anti-tumor or anti-cancer-promoting activity (ref. 2).

Scheme 1

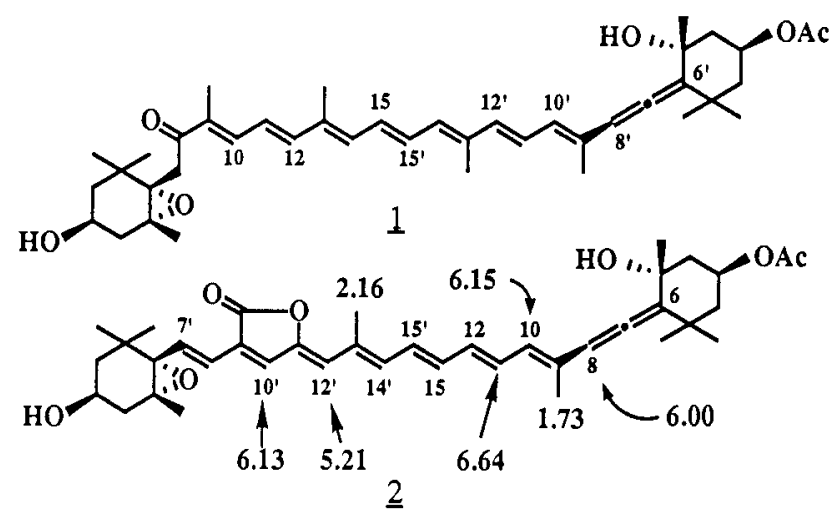

\section{PERIDININ}

\section{Photoisomerization of peridinin}

Although peridinin $\underline{2}$ has an allenic bond in the main polyene chain, it is representative of the butenolide carotenoids. Methodology for its synthesis has been developed (refs. 3-6) and its total synthesis in the optically active form accomplished (refs. 4,7). As an extension of these studies, the photochemical behaviour of $\underline{2}$ was investigated (ref. 8). Direct irradiation (20 min) with a daylight fluorescent lamp (15W) of $2 \underline{2}$ in benzene solution containing a catalytic amount of iodine at room temperature produced the isomeric mixture, HPLC separation of which yielded $\underline{3}, \underline{4}, \underline{5}$ and $\underline{2}$ in the proportions of $c a$. 1:4:3:8 (Scheme 2). Isomers $\underline{3}$ and $\underline{4}$ were determined to be $11 ' E$ 
and $9 Z$ isomers respectively, on the basis of their ${ }^{1} \mathrm{H}-\mathrm{NMR}$ data. The isomer $\mathbf{5}$ was assumed to be an allenic isomer $(6 S)$ of $\underline{2}$ from the chemical shift $(86.10)$ of $\mathrm{H}-8$. Confirmation of its structure was given by chemical synthesis.

Scheme 2

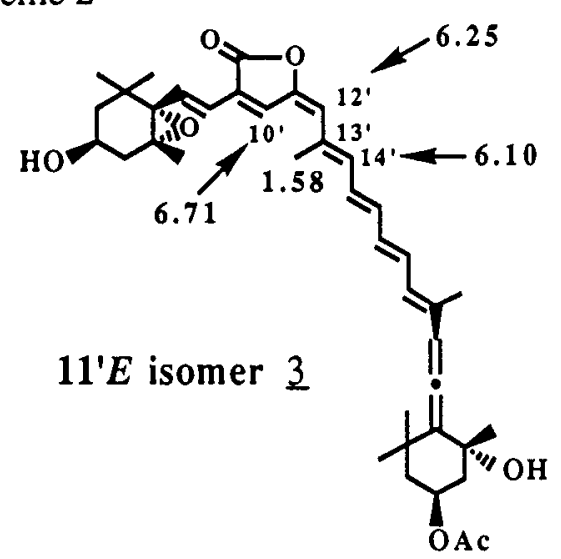

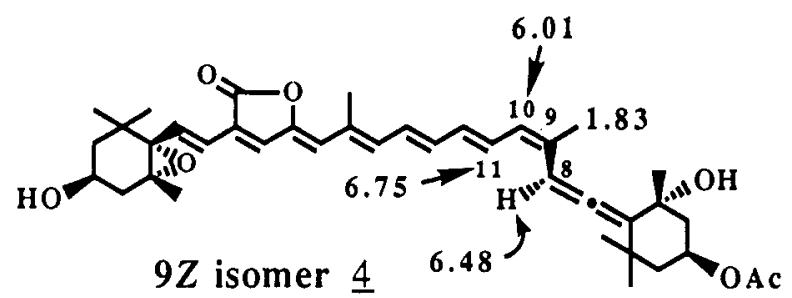

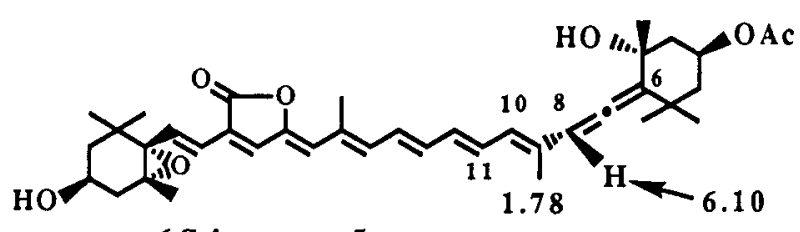

\section{Synthesis of $(6 S)$-peridinin}

Direct irradiation [(benzene solution, daylight fluorescent lamp15W), 2h] of the $6 R$ allenic sulfone $\underline{6}$, the important intermediate in the first total synthesis of optically active 2 , resulted in the remarkable photoisomerization of an allenic double bond and provided a 1:1 mixture of $\underline{6}$ and 7 (Scheme 3 ). The chirality of $I$ was chemically proved by ozonolysis to the allenic ketone $\underline{8}$, whose spectral data including optical properties were identical with those of an authentic specimen prepared according to the literature (ref. 9). By the same methodology as applied in the synthesis of $(6 R)$ peridinin 2 , the $\alpha$-sulfonyl carbanion prepared from the $6 S$ allenic sulfone $\underline{I}$ and LDA was treated with 9 at $-78^{\circ} \mathrm{C}$ to yield the condensed products, which were purified by preparative HPLC in the dark to furnish $(6 S)$-peridinin 5 and its $11^{\prime} E$ isomer, respectively, in pure form. The spectral properties of synthetic 5 were in good agreement with those of the isomer 5 isolated from the photoisomerization mixture of $\underline{2}$.

\section{Scheme 3}
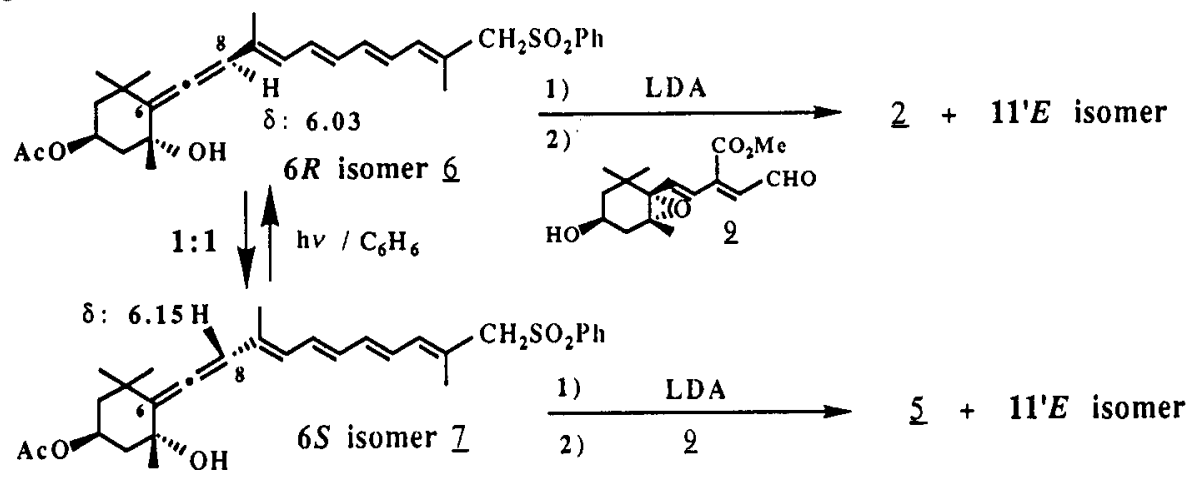

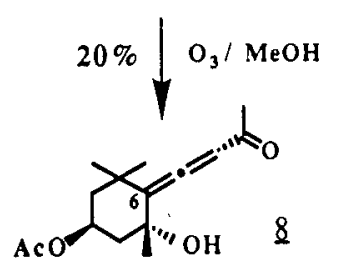


(6S)-Peridinin 5 was also isomerized in benzene solution by irradiation in the presence of iodine. In addition, $S$ to $R$ isomerization of the allenic bond in the $6 S$ allenic sulfone 7 was observed under the same irradiation conditions as in the case of $\underline{6}$. Thus, the photochemical behaviour of $\underline{2}, \underline{5}, \underline{6}$, and $\underline{I}$ suggests that, in the allenic carotenoids, isomerization around the allenic bond or its neighbouring bond occurs predominantly and supports the proposed biosynthetic mechanism for the allenic carotenoids (ref. 10).

\section{FUCOXANTHIN}

Fucoxanthin 1 is known to be widely distributed in the brown algae and to function as a light-harvesting pigment for photosynthesis. Although ca.17 years have passed since the absolute stereostructure of 1 was determined (ref. 11), there has been no report on synthetic studies of 1 , probably because of difficulties in constructing the $\beta, \gamma$-epoxy ketone, conjugated with the polyene, which was known to be extremely labile to alkali (ref. 1). Therefore, synthesis of 1 is a fascinating challenge for the organic chemist. The first total synthesis of optically active fucoxanthin was achieved according to the building principle $\mathrm{C}_{15}$ (A part) $+\mathrm{C}_{10}+\mathrm{C}_{15}(\mathrm{~B}$ part $)=\mathrm{C}_{40}$ as shown in Scheme 4 .

Scheme 4

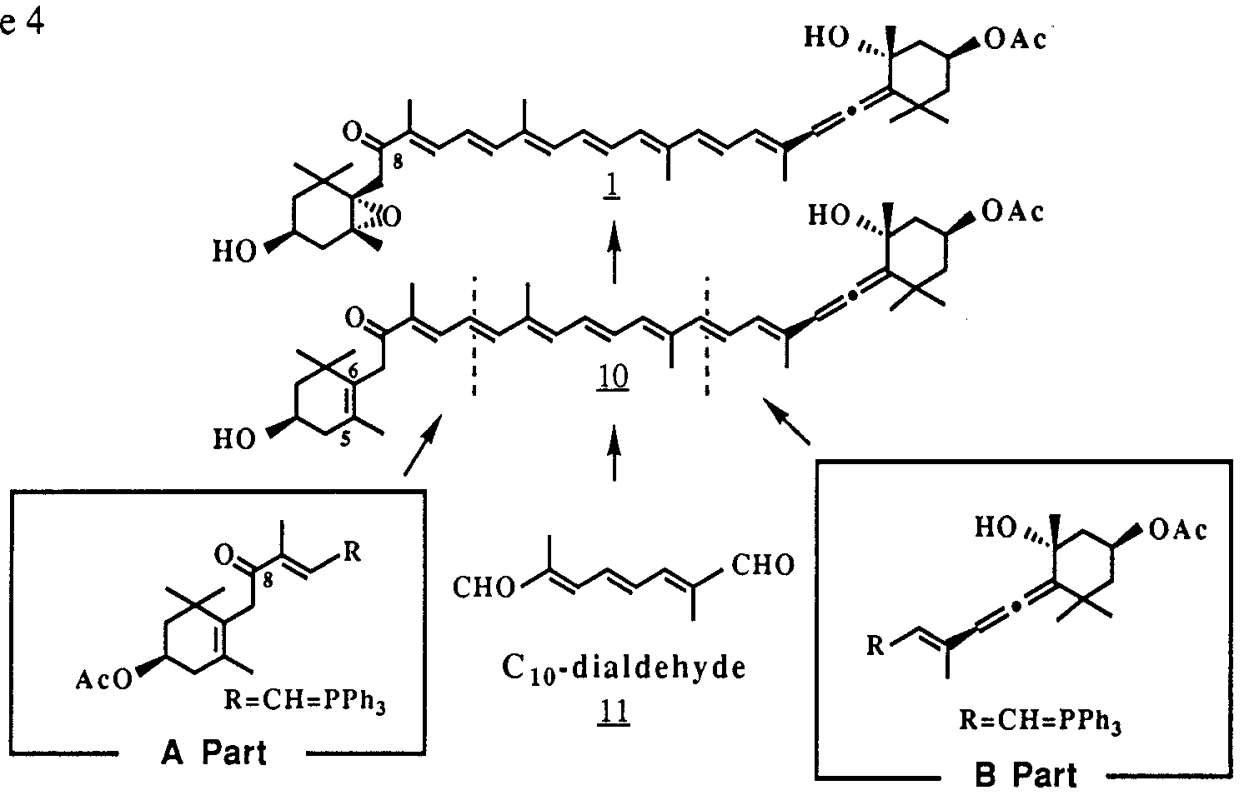

\section{Synthesis of the $\mathrm{C}_{15-8-0 \times 0}$ compound}

The A part was constructed by the application of the key reaction, i.e. the rearrangement of $\alpha$-acetylenic alcohols to $\alpha, \beta$-unsaturated carbonyl compounds by silylvanadate catalyst (ref. 12,13) and subsequent iodine-catalyzed isomerization $(\alpha, \beta$ unsaturated to $\beta, \gamma$-unsaturated ketones). The known $C_{15}-\alpha$-acetylenic alcohol 13 (ref. 7 ), the intermediate in the synthesis of 2 , was prepared in an optically active form ( $97 \%$ ee) starting from the readily available $(4 R, 6 R)$-4-hydroxy-2,2,6-trimethylcyclohexanone 12 (Scheme 5). 
Scheme 5<smiles>C[R16]O[C@@H]1C[C@@H](C)C(=O)C(C)(C)C1</smiles>

12
1)

$\mathrm{Li}$

2) $\mathrm{p} \cdot \mathrm{TsOH} / \mathrm{MeOH}$

3) $\mathrm{Ac}_{2} \mathrm{O} / \mathrm{Py}$

4) recrystallization

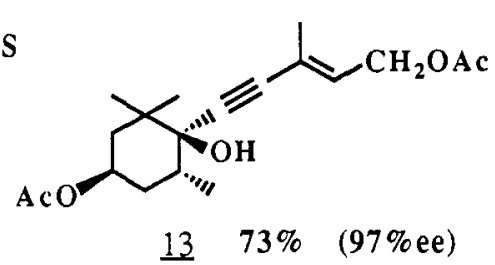

$13 \quad 73 \% \quad(97 \%$ ee $)$

Reaction of the $C_{15}-\alpha$-acetylenic alcohol 13 with tris(triphenylsilyl)vanadate/ triphenylsilanol in refluxing xylene, containing a small amount of benzoic acid, afforded the $\alpha, \beta$ - and $\beta, \gamma$-unsaturated ketones $\underline{14}$ (35\%) and $\underline{15}$ (58\%). Their structures were determined on the basis of the IR and ${ }_{1} \mathrm{H}-\mathrm{NMR}$ data including NOE experiments. The $6 Z$ isomer 14 was transformed into the $\beta, \gamma$-unsaturated isomer 15 in $80 \%$ yield by treatment with iodine in refluxing heptane (Scheme 6 ).

Scheme 6

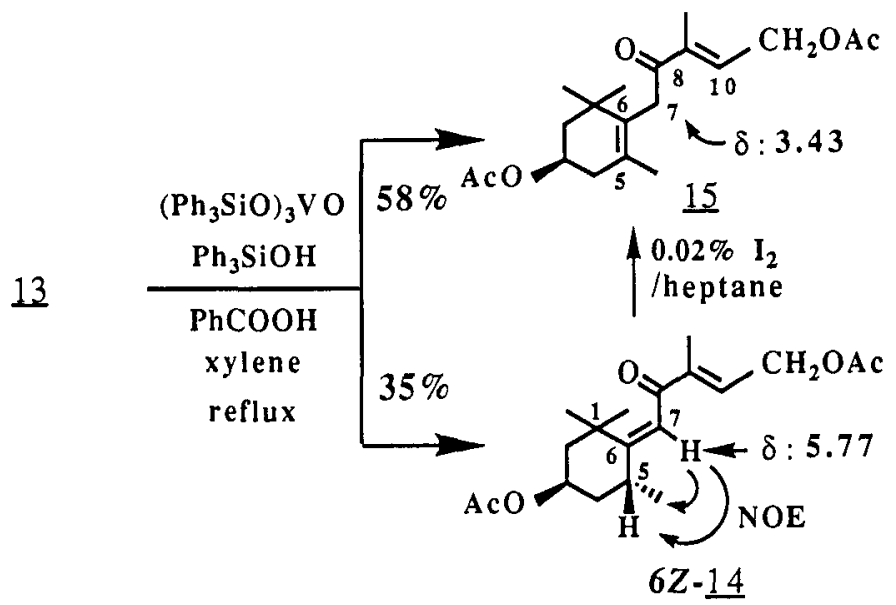

Mild hydrolysis of the ketone 15 with $10 \% \mathrm{~K}_{2} \mathrm{CO}_{3}$ gave in quantitative yield the hydroxy-enone 16 which was reacted with $\mathrm{LiCl}-\mathrm{MsCl}$ followed by treatment with $\mathrm{PPh}_{3}$

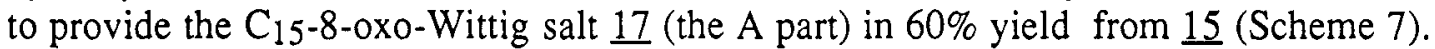

Scheme 7

15

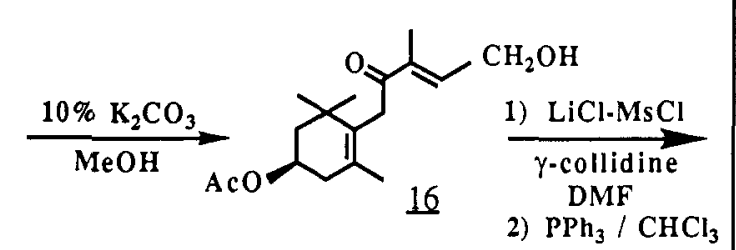

reflux

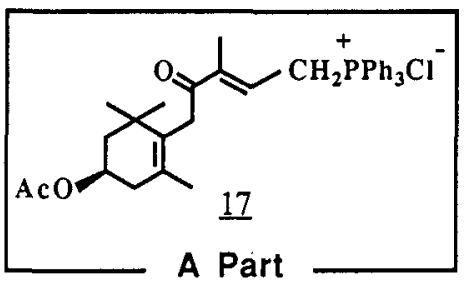

$60 \%$ from 15

\section{Sunthesis of $C_{15}$-allenic phosphonium chloride}

The $\mathrm{C}_{15}$-acetylenic diacetate $\underline{13}$ was transformed in four steps into the known allenic dihydroxy aldehyde $\underline{18}$ (ref. 7) which, by acetylation and subsequent $\mathrm{NaBH}_{4}$-reduction, was converted into the allenic alcohol 19 in $77 \%$ yield. Treatment of 19 with LiCl$\mathrm{MsCl}$ and successive reaction with $\mathrm{PPh}_{3}$ gave the $\mathrm{C}_{15}$-allenic phosphonium chloride 20 (the B part) in $73 \%$ yield (Scheme 8 ). 
Scheme 8<smiles>CC(=O)OCC=C(C)C#C[C@@]1(O)C[C@H](O)C[C@@H](OC(C)=O)C1</smiles>

$\underline{13}$

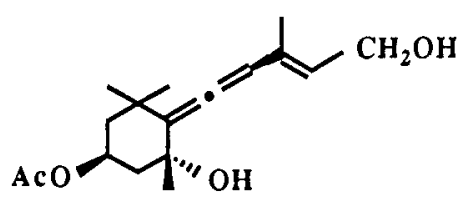

19

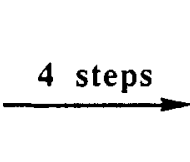

1) $\mathrm{LiCl}-\mathrm{MsCl}$

2) $\mathrm{PPh}_{3} / \mathrm{CHCl}_{3} / 50^{\circ} \mathrm{C}$

$73 \%$

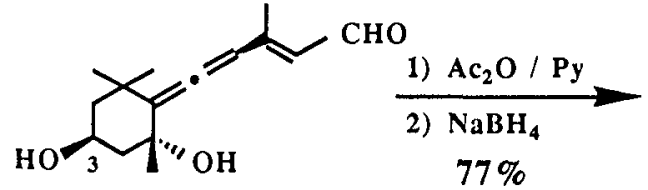

$\underline{18}$

$77 \%$

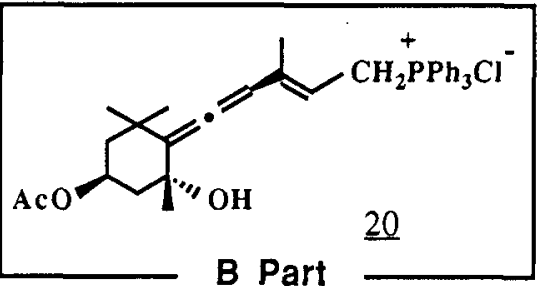

\section{Synthesis of ontically active fucoxanthin}

The Wittig condensation of 17 with $\mathrm{C}_{10}$-dialdehyde 11 in the presence of $\mathrm{NaOMe}$ as base and followed by hydrolysis $(5 \% \mathrm{NaOH})$ afforded a mixture of (all-E)-8-oxoapocarotenal $\underline{21}(32 \%)$ and the $11 Z$ isomer $\underline{22}(29 \%)$. These were cleanly separated in pure form. The latter was isomerized to the former in $94 \%$ yield by treatment with $\mathrm{PdCl}_{2}\left(\mathrm{CH}_{3} \mathrm{CN}\right)_{2} / \mathrm{Et}_{3} \mathrm{~N}$ in $\mathrm{CH}_{3} \mathrm{CN}$ (Scheme 9). Both 8-oxo-apocarotenals were characterized by UV-VIS, IR, and ${ }^{1} \mathrm{H}-\mathrm{NMR}$ spectral data. NOE experiments showed that the 8,9-single bond in 21 is s-trans.

Scheme 9

$\underline{17}$

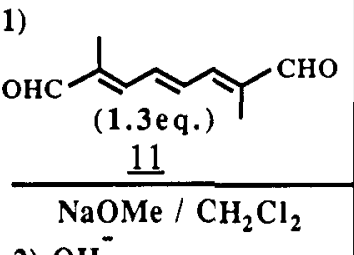

2) $\mathrm{OH}^{-}$

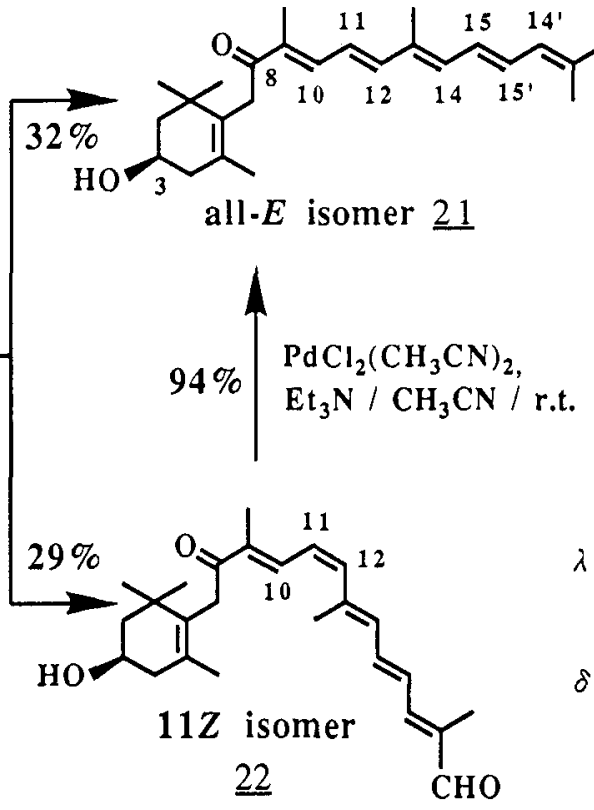

$\lambda: 289(\varepsilon 19,800)$

$393(\varepsilon 50,300)$

$408(\mathrm{sh})(\varepsilon 43,600)$

$\delta: 7.69$ (br d,J $=11.5,10 \cdot \mathrm{H})$

$6.46(t, J=11.5,11-\mathrm{H})$ 
After protection (TESOTf $/ \gamma$-collidine) of the hydroxyl group of $\underline{21}$, the product $\underline{22}$ was condensed with the $\mathrm{C}_{15}$-allenic phosphonium chloride 20 with $\mathrm{NaOMe}$ as base to give a mixture of the condensed products which was acetylated and desilylated with $(n-\mathrm{Bu})_{4} \mathrm{NF}(\mathrm{TBAF}) / \mathrm{AcOH}$ to provide a mixture (ca. 1:1) of the all- $E$ fucoxanthinskeleton compound $\underline{10}$ and its $11^{\prime} Z$ isomer $\underline{23}$. The separated products $\underline{10}$ (21\%) and $23(25 \%)$ were characterized by spectral data, respectively. Isomerization of the $11^{\prime} Z$ isomer 23 in the presence of $\mathrm{PdCl}_{2}\left(\mathrm{CH}_{3} \mathrm{CN}\right)_{2} / \mathrm{Et}_{3} \mathrm{~N}$ in $\mathrm{CH}_{3} \mathrm{CN}$ afforded the all- $E$ isomer 10 in $45 \%$ yield. Finally, the latter was epoxidized with MCPBA to provide a mixture of the syn-epoxide 24 and the anti-epoxide 25 in $36 \%$ yield in a ratio of $7: 2$ (Scheme 10). Spectral data (IR, UV-VIS, 1 H-NMR and MS), including CD data of the purified anti-epoxide 25 , were identical with those of natural fucoxanthin 1 . This is the first total synthesis of the optically active fucoxanthin.

Scheme 10
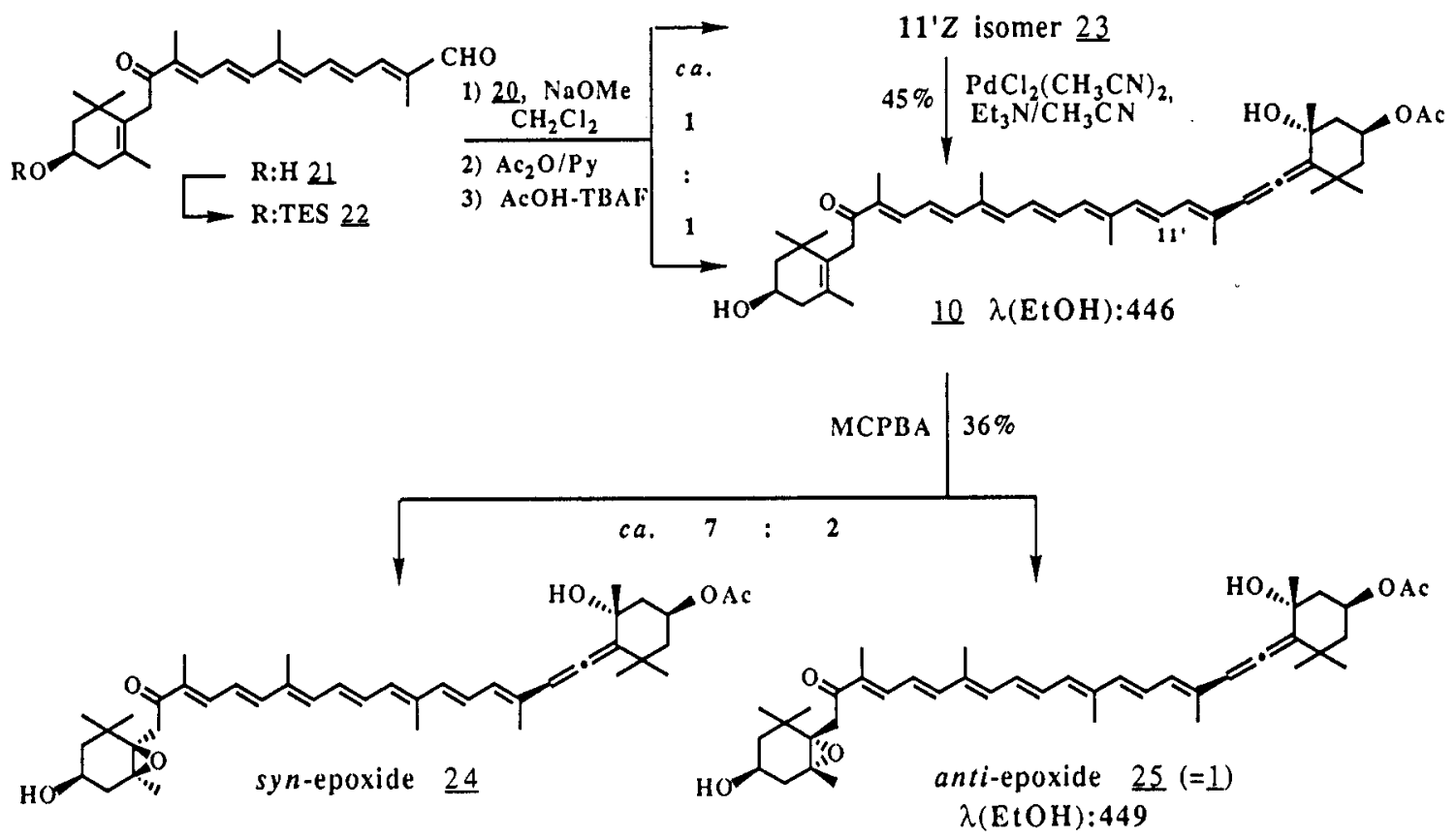

\section{Synthesis of fucoxanthin analogues}

In relation to studies on the effect of molecular structure on the relaxation processes of carotenoids containing a carbonyl group, four fucoxanthin analogues $\underline{26}, \underline{27}, \underline{28}$, and $\underline{29}$ (Scheme 11) were prepared by the Wittig condensation: repeated HPLC in the dark of the condensed mixture in the final step gave the $E$ and $Z$ isomers in pure form. Their structures were characterized by $500 \mathrm{MHz}{ }^{1} \mathrm{H}-\mathrm{NMR}$ data. From their $\lambda$ max values compared with those of 1 and 10 it has been found that the allenic part in 1 corresponds to one double bond of the conjugated polyenes. 


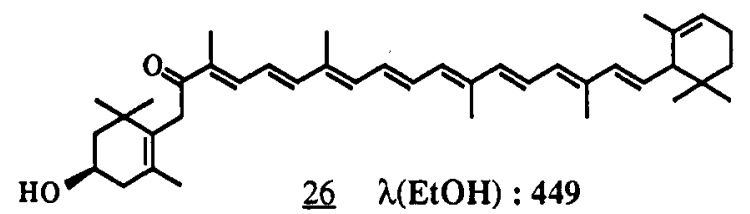<smiles>CC(=O)/C=C/C=C(C)\C=C\C=C\C=C(C)\C=C\C=C(C)\C=C\C1C(C)=CCCC1(C)C</smiles>

$28 \quad \lambda(\mathrm{EtOH}): 447$<smiles>[Z10][X]c1ccccc1</smiles><smiles>CC=CC(C)=CC=CC(C)=CC=CC=C(C)C=CC=CC(C)=CC=C(C)C</smiles>

$\underline{29} \lambda(\mathrm{EtOH}): 447$

\section{RETINOIDS}

For the investigation of the conformation of the chromophore around the trimethyl cyclohexene ring and of the origin of the induced $\beta$ circular dichroism band in rhodopsin, three kinds of $\mathrm{C}_{6}-\mathrm{C}_{7}$ single bond-fixed retinal analogues $\underline{30}, \underline{31}$ and $\underline{32}$ were synthesized in the $11 Z$ form (refs. 14-16). The UV-VIS, CD data and opsin shift of the new rhodopsin analogue derived from $\underline{32}$ were very close to those of native rhodopsin. This is the first time that the torsional angle around the 6-7 single bond in the rhodopsin chromophore has been chemically substantiated by using a $6 \mathrm{~s}$-fixed bicyclic retinal analogue.

Scheme 12
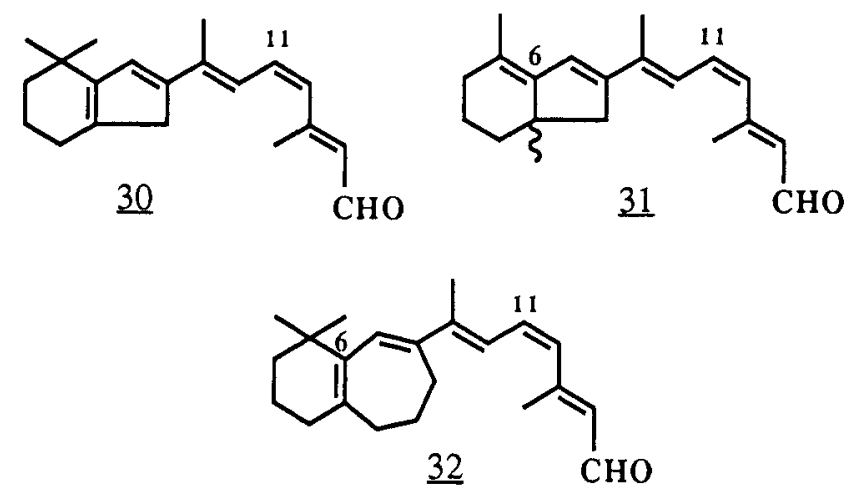

\section{ACKNOWLEDGEMENT}

The authors are indebted to Professor Y. Koyama, Kwansai Gakuin Unversity, and Professor K. Tsujimoto, University of Electro-Communications, for their invaluable gift of natural fucoxanthin. This work was supported in part by research grants from both the Science Research Promotion Fund of the Japan Private School Promotion Fundation and the Ministry of Education, Science, and Culture (Japan). We also appreciate the financial and chemical support of Kuraray Co., Ltd. Japan. 


\section{REFERENCES}

1. S. Liaaen-Jensen, Pure Appl. Chem., 63, 1-12 (1991).

2. H. Nishino et al, Abstr. 10th Int. IUPAC Carotenoid Symp. P9-16, Trondheim, 1993.

3. M. Ito, Y. Hirata, Y. Shibata and K. Tsukida, J. Chem. Soc.. Perkin Trans. 1, 197-199 (1990).

4. M. Ito, Pure Appl. Chem., 63, 13-22 (1991).

5. M. Ito, Y. Yamano and Y. Shibata, in Methods Enzymol., 213, 13-22 (1992).

6. M. Ito, Y. Katsuta, Y. Yamano and K. Tsukida, J. Chem. Soc.. Perkin Trans. 1, 987-993 (1993).

7. Y. Yamano and M. Ito, J. Chem. Soc., Perkin Trans. 1, 1599-1610 (1993).

8. Y. Yamano, S. Sumiya, K. Suzuki, Y. Kurimoto, Y. Koyama, T. Shimamura and M. Ito, Tetrahedron Lett., 33, 2991-2994 (1992).

9. A. Baumeler, W. Brade, A. Haag and C. H. Eugster, Helv. Chim. Acta, 73, 700715 (1990).

10. S. Isoe, S. Katsumura, S. B. Hyeon and T. Sakan, Tetrahedron Lett., 10891092 (1971).

11. K. Bernhard, G. P. Moss, G. Tóth and B. C. L. Weedon, Tetrahedron Lett., 115118 (1976).

12. H. Pauling, D. A. Andrews and N. C. Hindley, Helv. Chim. Acta, 59, 1233-1243 (1976).

13. G. L. Olson, H. C. Cheung, K. D. Morgan, R. Borer and G. Saucy, Helv. Chim.

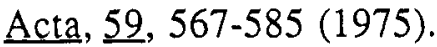

14. M. Ito, Y. Katsuta, M. Imamoto, Y. Shichida and T. Yoshizawa, Photochem. Photobiol., 56, 915-919 (1992).

15. Y. Katsuta, M. Sakai and M. Ito, J. Chem. Soc.. Perkin Trans. 1, in press.

16. A. Wada, M. Sakai, M. Imamoto, Y. Shichida, T. Yoshizawa and M. Ito, Chem. Pharm. Bull., 41, 793-795 (1993). 